\title{
On the Improved Correlative Prediction Scheme for Aliased Electrocardiogram (ECG) Data Compression
}

\author{
Xin $\mathrm{Gao}^{1}$, Member, IEEE \\ 1: Department of Electrical and Computer Engineering, The University of Arizona, Tucson, AZ85721, USA \\ E-mail:xgao1985@email.arizona.edu
}

\begin{abstract}
An improved scheme for aliased electrocardiogram (ECG) data compression has been constructed, where the predictor exploits the correlative characteristics of adjacent QRS waveforms. The twin- $R$ correlation prediction and lifting wavelet transform (LWT) for periodical ECG waves exhibits feasibility and high efficiency to achieve lower distortion rates with realizable compression ratio (CR); grey predictions via GM $(1,1)$ model have been adopted to evaluate the parametric performance for ECG data compression. Simulation results illuminate the validity of our approach.

Index Terms: Data compression, correlative prediction, electrocardiogram (ECG), lifting wavelet transform (LWT), GM $(1,1)$ model
\end{abstract}

\section{INTRODUCTION}

The dynamic system for monitoring and extracting electrocardiogram (ECG) signals or images has been demonstrated for extensive applications in heart disease diagnose. Since the complete ECG data sequence for at least successive 24-hour must be recorded for each patient, enormous storage equipment can be compulsory for that the sampled ECG data urges durable maintenance; e.g., in the double-channel Holter system [1], a memory of 82 Mega bits must be taken up for the daily sampled ECG data storage by the resolution of 11 bits and $360 \mathrm{~Hz}$ for sampling rate per channel. To restore data efficiently and minimize redundancy at the penalty of loseless or low lossy cost, a variety of versatile techniques for data compression [1]-[5] have been established for both research orientation and practical design. In the past decade, technical categories as time-domain compression (e.g., polynomial fitting [2] or predictive coding [5], [7]) and orthogonal transform-domain compression [3]-[4], [6], [8], [10] had been established. Efficient data compression techniques favor correlative models and generally take advantage of the correlations between adjacent QRS waves for sequential prediction.

Utilizing the correlations between adjacent QRS waves of mixed noisy electrocardio-signals, an improved scheme for ECG data compression has been put forward to predict the minimum mean-square error (MMSE), then step further to perform integer wavelet transform, quantization, run-length coding, and arithmetic coding to achieve ECG data compression. The compressed data reaches considerably high compression ratio (CR) while still carries most useful message for ECG signals; meanwhile, a feasible lower distortion is maintained by the proposed method in contrast to Sabah's [1]. Grey prediction on the basis of GM $(1,1)$ model is provided after the simulated trials.

\section{PROBLEM FormULATION}

The adult ECG waveform for a complete normal period [2], [6] is depicted in Fig. 1, which consists of $P, T$ waves and the central QRS interval. The message that carried by ECG waveform reveals heart rate, amplitude and duration for heart pulse or earlier symptoms of heart disease. Exploiting the correlativity between QRS waveforms suggests an efficient technique for ECG data compression.

\section{A. Correlative predictions between QRS waveforms}

In contrast to the MMSE principle and conventional predictive coding [4], the predictor above could not be compatible for non-stationary signals such as ECG, considerably large prediction errors can be generated and reached a maximum value especially when the signal amplitude depicts fast fluctuation. We exploit the close relativity in the successive phases between two adjacent QRS waveforms; hence, a sequential data of $p$ points of the former QRS waveform is adopted to predict the present waveform and thus level down the prediction errors. The predictor output is expressed as [4]:

$$
y(n)=\sum_{i=1}^{p} \alpha_{i} x(n-i-T)
$$

And the prediction error $\varepsilon(n)$ can be estimated as

$$
\varepsilon(n)=x(n)-y(n)=x(n)-\sum_{i=1}^{p} \alpha_{i} x(n-i-T)
$$

where the adjacent $p$ points stands for the orders of correlative predictor, of which the coefficients $\alpha_{i}$ can be obtained by Yule-Walker equations, $T$ represents the time-interval between adjacent $R$-waves ( $R$ - $R$ interval).

Since the prediction is processed between two adjacent QRS waveforms, it can be named as twin- $R$ correlative prediction. Consider the energy $E_{p}$ of prediction errors as

$$
E_{p}=E\left[\varepsilon^{2}(n)\right]=E\left\{\left[x(n)-\sum_{i=1}^{p} \alpha_{i} x(n-i-T)\right]^{2}\right\}
$$

where the expanded formula can be viewed as

$$
\begin{aligned}
E_{p} & =E\left\{x^{2}(n)-2 \sum_{i=1}^{p} \alpha_{i} x(n) x(n-i-T)+\sum_{i=1}^{p} \alpha_{i} \sum_{j=1}^{p} \alpha_{j} x(n-i-T) x(n-j-T)\right\} \\
& =R(0)-2 \sum_{i=1}^{p} \alpha_{i} R(i+T)+\sum_{i=1}^{p} \alpha_{i} \sum_{j=1}^{p} \alpha_{j} R(j-i)
\end{aligned}
$$

Practically, the correlation coefficient is computed by

$$
R(m)=\frac{1}{N} \sum_{n=1}^{N-1} x(n) x(n-m)
$$

Let $\frac{\partial E_{p}}{\partial \alpha_{i}}=0$ to obtain a minimum for $E_{p}(m=0,1, \ldots, p-1)$, 


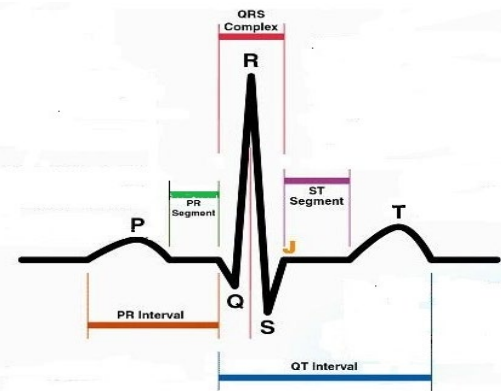

Fig. 1. The periodic waveform sketch for an adult's ECG

Equation (4) can be simplified as

$$
\sum_{i=1}^{p-1} \alpha_{i} R(m-i)=R(m+T) \quad i=0,1, \ldots, p-1
$$

Combining the equations for the matrix we constructed,

$$
\left[\begin{array}{cccc}
R(0) & R(1) & \mathrm{L} & R(p-1) \\
R(1) & R(0) & \mathrm{L} & R(p-2) \\
\mathrm{M} & \mathrm{M} & \mathrm{O} & \mathrm{M} \\
R(p-1) & R(p-2) & \mathrm{L} & R(0)
\end{array}\right]\left[\begin{array}{c}
\alpha_{0} \\
\alpha_{1} \\
\mathrm{M} \\
\alpha_{p-1}
\end{array}\right]=\left[\begin{array}{c}
R(T) \\
R(T+1) \\
\mathrm{M} \\
R(T+p-1)
\end{array}\right]
$$

Solving Eq. (7) yields the coefficients of $\alpha_{i}$.

\section{B. Lifting Wavelet Transform (LWT) to compress ECG}

Referring to the second-generation methods for wavelet construction [6], LWT (also known as integer-to-integer wavelet transform) is regarded as a strong implementation collaborating with a few algorithms of integer square zero-tree wavelet coding. Typically, the lift scheme of LWT has 3 steps: splitting, predicting and updating [6]:

To begin with, the ECG data sequence $\left\{e_{j}\right\}$ is splitted into two sequences $\left\{o_{j-1}\right\}$ and $\left\{e_{j-1}\right\}$ which represent odd and even numerals, respectively. This step is denoted as

$$
\operatorname{split}\left(e_{j}\right)=\left(e_{j-1}, o_{j-1}\right)
$$

Utilizing the correlativity between QRS waves, the odd sequence $\left\{o_{j-1}\right\}$ is predicted throughout the predictor filter group $P$ by the earlier even sequence $\left\{e_{j-1}\right\}$ such as

$$
o_{j-1}:=o_{j-1}-P\left(e_{j-1}\right)
$$

The updating step indicates that a better subset $\left\{e_{j-1}\right\}$ urges construction holds some integral characteristics that of integrity for the original $\left\{e_{j}\right\}$. Hence, an updating filter $U$ is adopted by exploiting the discrepancy between a certain parameter (mean, variance or wavelet vanishing moments [4], [6]) and $\left\{e_{j}\right\}$, by which we have

$$
e_{j-1}:=e_{j-1}+U\left(o_{j-1}\right)
$$

Similarly, with respect to (8)-(10), the inverse transform of LWT for signal reconstruction can be obtained by

$$
\left\{\begin{array}{c}
e_{j-1}:=e_{j-1}-U\left(o_{j-1}\right) \\
o_{j-1}:=o_{j-1}+P\left(e_{j-1}\right) \\
e_{j}=\operatorname{Merge}\left(e_{j-1}, o_{j-1}\right)
\end{array}\right.
$$

Applying $(4,2)$ LWT to decompose and reconstruct ECG signals, the suggested iterations are represented as [5], [7]:

$$
\left\{\begin{array}{l}
\left.o_{j}[n]:=e_{j-1}[n]+\left\lfloor\frac{1}{16}\left\{\left(e_{j-1}[n+2]\right)-9\left(e_{j-1}[n+2]\right)+e_{j-1}[n-1]\right)+e_{j-1}[n-1]\right\}+\frac{1}{2}\right\rfloor \\
e_{j}[n]:=e_{j-1}[n]+\left\lfloor\frac{1}{4}\left(o_{j}[n]+o_{j}[n-1]\right)+\frac{1}{2}\right\rfloor
\end{array}\right.
$$

where $\lfloor$.$\rfloor denotes the execution of round-off operation.$
In contrast to other wavelet-transform methods [4], [5], advantages of LWT can be summarized as follows:

(i) By LWT, we perform direct operation of odd-even splitting for ECG so that the dependence comes down for the down-sampling of lowpass and highpass signal components; the inverse LWT can be easily achieved via adjusting the positive/negative operator of LWT;

(ii) Floating point operation can be avoided to reduce operation times due to the integer coefficients; also, since it is only reliable to the operation of addition and shift, the practical hardware circuits can be easier for realizations;

(iii) The quality for signal recovery has no relevance to whatever style of boundary continuation.

\section{THE UNIFIED METHOD FOR ECG DATA COMPRESSION AND PARAMETER PREDICTION}

\section{A. Twin-R Correlation Predictor for ECG compression}

In this subsection, we propose the framework for improving ECG compression via correlation predictor, which can be decomposed into several procedures. Let the implement $D$ be the first-order time delay, $P_{i}$ be the location of the $i$ th $R$-wave. It is also denoted that $\mathbf{A}_{j}=\left\{\alpha_{j, 0}, \alpha_{j, 1}, \ldots, \alpha_{j, p-1}\right\}$ representing the aggregation of predictor coefficients for the twin- $R$ interval of the $j$ th ECG sequence. Our scheme is derived by the steps as follows [5]:

Step 1) First-order prediction for the original ECG signal with length $N$ so as to reduce the DC components of signals; the redundancy within smooth district of the ECG samples calls for elimination can be formulated as:

$$
y(n)=x(n)-x(n-1), n=0,1, \ldots, N-1 .
$$

Step 2) Identify $P_{i}$, the locations of $R$-wave for each QRS waveform, and compute $T_{i}$ (twin- $R$ time-interval) between adjacent $R$-waves. Obviously we have $T_{i}=P_{i+1}-P_{i}$. With reference to Daskalov's algorithm [6], Step 2 combines advantages of accelerating higher recognition rate and operation speed; negative effects such as noise interference or baseline shift are also tolerated.

Step 3) Calculate the central position of adjacent twin- $R$ waves, for which $m_{i}=\left(P_{i+1}+P_{i}\right) / 2$.

Step 4) Correlation prediction for $y(n)$ by Eq.(2):

$$
\begin{aligned}
d(n) & =y(n)-\sum_{k=0}^{p-1} \alpha_{j, k} y\left(n-i-T_{i-1}\right), m_{i}-l \leq m_{i}+l, \\
& l=\min \left(m_{i-1}-m_{i-2}, m_{i}-m_{i-1}\right) / 2, i=1, \ldots, N_{j} .
\end{aligned}
$$

where $p$ and $N_{j}$ represent the predictor order and the $R$-wave counts of the $j$ th ECG data sequence, respectively. ( $p=4$ is adopted for generality) The $k$ th prediction coefficient $\alpha_{j, k}$ of ECG signal can be obtained by (8), of which the notation is 16-bit in the compressed data sequence. Considering slow drift for the pattern of QRS form, the data stream is partitioned by an interval of 30 seconds, while the same predictors are implemented for the continuous QRS waveforms of the same ECG data block. In that case, we have reduced the computational loads and increased the speed for compression at modest efficiency cost of data compression.

Step 5) The four-level LWT [3] for prediction error signal $d(n)$ is now carried out via (13), where the length of $w(n)$ remains $N$ after transform. $w(n)$ consists of the subband signal group $\left\{o_{i}(n)=1,2,3,4\right\}$ and the approximated signal $e_{4}(n)$, 
whose length are constructed as $N / 2, N / 4, N / 8, N / 16$ and $N / 16$, respectively.

Step 6) Scalar quantization and run-length coding [5] for $w(n)$. Since quite a few zero-coefficients come up after quantization toward $w(n)$, run-length coding for these successive zeros offers an effective tool for shortening ECG data sequence. Variable quantization coefficients could be selected due to the distributed importance of multi-scale subband signals in different districts.

Step 7) Arithmetic coding [5] for the run-length encoded data sequence, after which the data block is merged by three parts: the constructed bit streams, vector $P_{i}$ for $R$-wave localization and the twin- $R$ predictor coefficients $\mathbf{A}_{j}=\left\{\alpha_{j, 0}\right.$, $\left.\alpha_{j, 1}, \ldots, \alpha_{j, p-1}\right\}$ for each sequence.

Up to now, we have proposed the schemed framework to compress ECG data sequence. Since the decompression algorithm can be built up by the reversed procedure of the listed steps above, in order to observe the correlativity and the fluctuation tendency between two sequential data, a Lemma can be put forward to construct the Grey model [9]:

Lemma 1 [9]: A stationary sequence $T_{0}=\left\{T_{0}(k) \mid k=1, \ldots, n\right\}$ $=\left\{T_{0}(1), \ldots, T_{0}(n)\right\}$ is considered for reference, where $k$ stands for the time point. Assume a number of $m$ sequences are observed for comparison, $T_{\mathrm{i}}=\left\{T_{\mathrm{i}}(k) \mid k=1,2, \ldots, n\right\}=\left\{T_{\mathrm{i}}(1)\right.$, $\left.T_{\mathrm{i}}(2), \ldots, T_{\mathrm{i}}(n)\right\}, i=1,2, \ldots, n$. Define

$$
\xi_{k}=\frac{\min _{i} \min _{k}\left|T_{0}(k)-T_{i}(k)\right|+\rho \max _{i} \max _{k}\left|T_{0}(k)-T_{i}(k)\right|}{\left|T_{0}(k)-T_{i}(k)\right|+\rho \max _{i} \max _{k}\left|T_{0}(k)-T_{i}(k)\right|}
$$

as the correlation coefficient of comparison sequence with respect to the reference sequence $T_{0}$ at time $k$, where $\rho \in[0,1)$ represents the resolution coefficient, while also $\operatorname{minmin}_{i}\left|T_{0}(k)-T_{i}(k)\right|$ and $\max _{i} \max _{i}\left|T_{i}(k)-T_{0}(k)\right|$ stand for the minimum and maximum difference value between two-levels, respectively. In grey system theory [9], the relevance of sequence $T_{i}$ to original $T_{0}$ is defined as $r_{i}=\frac{1}{n} \sum_{k=1}^{n} \xi_{k}$. In general case, the geometry similarity for two sequences reflects the degree of correlativity.

The certain sequence $T_{\mathrm{i}}=\left\{T_{\mathrm{i}}(1), \quad T_{\mathrm{i}}(2), \ldots, \quad T_{\mathrm{i}}(n)\right\}$, $\bar{T}=\left(1, \frac{T(2)}{T(1)}, \cdots \frac{T(n)}{T(1)}\right)$ is denoted as the initialized sequence of original $T_{\mathrm{i}}$. Take the simplest case of $i=1,2$ for example

$$
T_{i}=\left(1, \frac{T_{i}(1)}{T_{i}(2)}, \frac{T_{i}(1)}{T_{i}(3)}, \cdots, \frac{T_{i}(1)}{T_{i}(k)}\right), i=1,2 ; k=N
$$

With respect to Lemma 1 , the degree of correlativity between can be obtained by solving Eq. (15) when taking the expectation of $\rho=0.5$; denote also

$$
\sigma_{i}=\sum_{k=1}^{N} k T_{i}(k)-\sum_{k=1}^{N} T_{i}(k) \sum_{k=1}^{N} \frac{k}{n}
$$

Determine whether the correlation factor is positive or negative. Positive relevance between $T_{1}$ and $T_{2}$ can be justified when $\operatorname{sign}\left(\sigma_{1} / \sigma_{n}\right) \operatorname{sign}\left(\sigma_{2} / \sigma_{n}\right)=1$; otherwise, it equals -1 for negative relevance. It is easy to compute $\sigma_{1}=1.1516, \sigma_{2}=$ -3.2976 ; the correlation factor $\sigma_{\mathrm{n}}$ is obtained by

$$
\sigma_{n}=\sum_{k=1}^{n} k^{2}-\left(\sum_{k=1}^{n} k^{2}\right) / n
$$

\section{B. Grey Model for Evaluative Parameter Prediction}

Based on the fundamental theory of grey prediction [9], GM $(1,1)$ model can be constructed to estimate the upcoming sequence number and evaluating the missed numerical values between time intervals. In this evaluation for the performance of ECG data compression, each parameter variable can be equalized as a time-sequence. Here we deduce the grey predictor as follows:

The differential equation in the form of whitening can be structured as $\frac{d T_{i}^{(1)}}{d t}+a T_{i}^{(1)}=u$, denote the estimate of $a$ as $\hat{a}=(a, u)^{T}$; least square (LS) update is shown as:

$$
\begin{aligned}
& \frac{d T_{i}^{(1)}}{d t}+a T_{i}^{(1)}=u, \quad \hat{a}=(a, u)^{T} \\
& \hat{\mathbf{a}}=\left(\mathbf{B}_{i}^{T} \mathbf{B}_{i}\right)^{-1} \mathbf{B}_{i}^{T} \mathbf{Y}_{1} \\
& \mathbf{B}_{i}=\left[\begin{array}{cc}
-\frac{1}{2}\left(T_{i}^{(1)}(1)+T_{i}^{(1)}(2)\right) & 1 \\
-\frac{1}{2}\left(T_{i}^{(1)}(2)+T_{i}^{(1)}(3)\right) & 1 \\
\mathrm{M} & \mathrm{M} \\
-\frac{1}{2}\left(T_{i}^{(1)}(n-1)+T_{i}^{(1)}(n)\right) & 1
\end{array}\right], \mathbf{Y}_{i}=\left[\begin{array}{c}
T_{i}^{(1)}(2) \\
T_{i}^{(1)}(3) \\
\mathrm{M} \\
T_{i}^{(1)}(n)
\end{array}\right]
\end{aligned}
$$

where $\mathbf{B}_{i}$ and $\mathbf{Y}_{i}$ correspondingly represent the data matrix and data vector of GM $(1,1)$ model.

The matrix equations above yield a general solution that

$$
T_{i}^{(1)}(k+1)=\left(T_{i}^{(0)}(1)-\frac{u}{a}\right) e^{a k}+\frac{u}{a}
$$

After the model parameters $(a, u)$ determined by respect to (19)-(21), the predictive values past or upcoming can be measured by this predictive $\operatorname{GM}(1,1)$ model.

Most notably, the grey model matches the extrapolate prediction which is variable corresponding to time shifts. Since the least sample only requires 4 adjacent continuous data points, the usefulness of $\operatorname{GM}(1,1)$ model in condition of scarce information and ambiguous system is apparently displayed and will be testified in Section IV.

\section{SimUlation RESUlts}

The simulated experiments have been conducted by using the database of mixed maternal electrocardiogram (MECG), fetal electrocardiogram (FECG) and the standard MIT-BIS Arrhythmia Database [1]. We first investigate a mixed ECG data sequence by comparing the predicted output of error signals. Fig. 2 depicts a data sequence (selected from time interval $[0: 1600]$ ) of extracted FECG signal via different predictors where Figs. 2(b)-(c) correspondingly did the first-order and fourth-order linear prediction, in contrast to Fig. 2(d) which constructed the twin- $R$ correlative prediction. It is indicated that the fourth-order linear predictor has smaller average errors than the first-order predictor while the false detection levels up; the twin- $R$ correlative predictor has the average errors considerably reduced other than the former two linear predictors [7].

The technique of LWT for compressing the ECG sequence [400:1000] after the presence of random noise by SNR = $10 \mathrm{~dB}$ has been displayed in Fig. 3. It is clear that the 
compressed data sequence in Fig. 3(b) maintained most details of the original

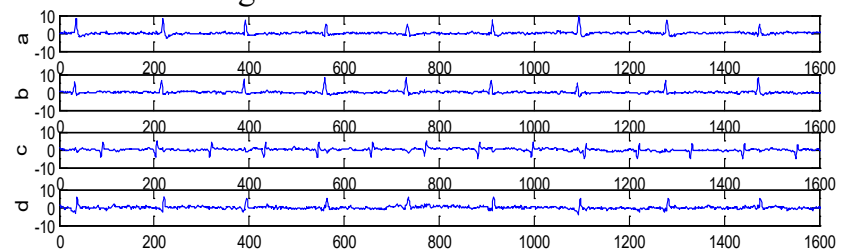

Fig. 2. (a) Originally detected FECG; (b) Sequential data output by single linear prediction; (c) by fourth-order linear prediction; (d) by twin- $R$ correlative prediction. (peak voltage denotes the location of $R$ waves)
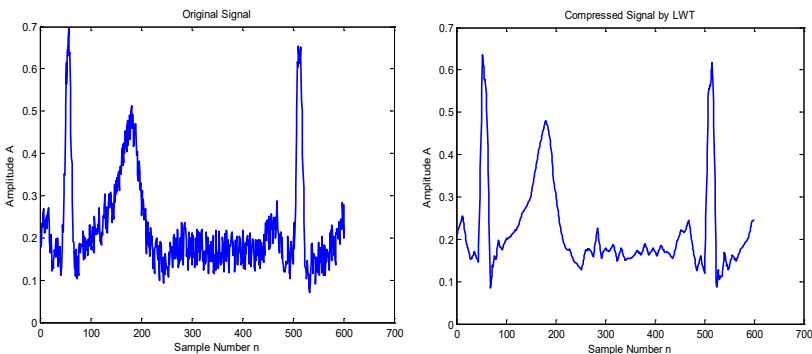

(a)

(b)

Fig. 3. (a) The ECG signal in [400:1000] after noise added (SNR $=10 \mathrm{~dB})$; (b) Compressed ECG signal by LWT

signal, while slightly energy loss has been exposed in the amplitude due to the round-off decomposition loss in LWT and quantization error.

The evaluative parameters for the scheme of ECG data compression are induced as the compression ratio (CR) and percent root mean-square difference (PRD) [6]. Without loss of generality, 24 cases out of 48 from database in [1] have been randomly selected as our testing sample in the following trial. By contrast to the algorithm of M. A. Sabah et al [1], sequential data compression has been trialed and the corresponding PRDs are also recorded with respect to different CRs (by altering quantization coefficients) in our approach. Table I shows the evaluated results for the obtained PRDs corresponding to $\mathrm{CR}$ where each numerical value of PRD has been averaged. It is suggested that the PRDs of our scheme are much smaller than that of Sabah's [1] when gaining the same CR; the unified approach of correlative prediction illustrates a lower distortion rate.

To obtain the predictive value of PRDs in accordance with the unknown CR for both methods, we construct the $\operatorname{GM}(1,1)$ for the "time-sequence" $T_{1}, T_{2}$ by concerning CR as time points and PRD as the sequential output. By (17) and (18), it is easy to justify that $T_{1}, T_{2}$ have positive correlativity. With respect to (19)-(22), the unknown coefficients and the solution to predictive $\operatorname{GM}(1,1)$ model are finally obtained as

$$
\begin{gathered}
T_{1}: T_{1}^{(1)}(k+1)=(2.10-328.91) e^{-0.0026784 k}+328.9096 \\
T_{2}: T_{2}^{(1)}(k+1)=(1.49-150.95) e^{-0.0049531 k}+150.9503
\end{gathered}
$$

The predicted PRDs for comparison of our scheme and the same reference are listed in Table II. For comprehensive views of the predictive methods, the derived GM $(1,1)$ model works well for the "extrapolated" time points and has much closer predicted results in contrast with real numerical values of PRD to CR [6]; on the other hand, the prediction for "interpolated" time points are more reliable to higher-order polynomial fittings [9], while functional fittings make less sense for extrapolated points corresponding to larger CR values.

TABLE I. COMPRESSION SCHEME FOR CR, PRD vs SABAH's METHOD [1]

\begin{tabular}{|c|c|c|c|c|c|c|}
\hline \multicolumn{2}{|c|}{ CR } & 2.0 & 3.0 & 5.0 & 7.0 & 9.0 \\
\hline \multirow{2}{*}{ PRD } & Sabah & 2.10 & 3.22 & 4.71 & 6.09 & 7.68 \\
\cline { 2 - 7 } & Ours & 1.49 & 1.81 & 2.75 & 3.78 & 5.01 \\
\hline \multicolumn{2}{|c|}{ CR } & 11.0 & 13.0 & 15.0 & 17.0 & 19.0 \\
\hline \multirow{2}{*}{ PRD } & Sabah & 9.33 & 11.10 & 12.31 & 14.04 & 16.78 \\
\cline { 2 - 7 } & Ours & 6.32 & 7.67 & 9.20 & 11.31 & 13.56 \\
\hline
\end{tabular}

TABLE II. PREDicted PRD (By GM(1,1) MODEL) VS SABAH's METHOD [1]

\begin{tabular}{|c|c|c|c|c|c|c|}
\hline \multicolumn{2}{|c|}{ CR } & 4.0 & 8.0 & 12.0 & 16.0 & 20.0 \\
\hline \multirow{2}{*}{ PRD } & Sabah & 3.85 & 7.31 & 10.74 & 14.12 & 17.48 \\
\cline { 2 - 7 } & Ours & 2.96 & 5.87 & 8.24 & 11.50 & 14.24 \\
\hline \multicolumn{2}{|c|}{ CR } & 21.0 & 23.0 & 25.0 & 27.0 & 29.0 \\
\hline \multirow{2}{*}{ PRD } & Sabah & 18.3 & 19.97 & 21.62 & 23.27 & 24.90 \\
\cline { 2 - 7 } & Ours & 14.91 & 16.25 & 17.58 & 18.90 & 20.23 \\
\hline
\end{tabular}

\section{CONCLUSION}

This paper has established an improved scheme that effectively exploits the correlation characteristics of time-domain ECG signals within adjacent twin- $R$ waves. The proposed method performs twin- $R$ correlation prediction and LWT for periodical ECG waves. The lower distortion rates in accordance with higher compression ratio indicates its feasibility of methodology; meanwhile, the cooperative grey model $\operatorname{GM}(1,1)$ stands up as a prospective technique for sequential data predicting and parameter evaluation.

\section{REFERENCES}

[1] M. A. Sabah, Al-Shrouf Anwer, and A-Z. Mohammed, "ECG data compression using optimal non-orthogonal wavelet transform," Med. Eng. \& Phy., vol. 22, pp. 39-46, 2000.

[2] W. Philips and G. De Jonghe, "Data compression of ECG's by high degree polynomial approximation," IEEE Trans. Biomed. Eng., vol. 39, no. 4, pp. 330-337, Apr. 1992.

[3] C. H. Ramiro, M. B. Velasco, F. C. Roldan, and F. P. Benito, "Efficient thresholding-based ECG compressors for high quality applications using cosine modulated filter banks," in 33rd Conf. IEEE Eng. Med. \& Biology Soci. (EMBS), Boston, USA, Aug.-Sep. 2011, pp. 7079-7082.

[4] S. M. S. Jalaleddine, C. G. Hutehens, R. D. Strattan, and W. A. Coberly, "ECG data compression techniques--a unified approach," IEEE Trans. Biomed. Eng., vol. 37, no. 4, pp. 329-343, Apr. 1990.

[5] A. Cohen and Y. Zigel, "Compression of multichannel ECG of multichannel long-term prediction," IEEE Eng. Med. \& Bio. Mag., vol. 17, no. 1, pp. 109-115, Jan.-Feb. 1998.

[6] Q. Lian, C. Wang, and Z. Hu, "An electrocardiogram compression algorithm based on correlative prediction of QRS interval," J. Electron. Meas. \& Ins., vol. 18, no. 1, pp. 29-33, Mar. 2004.

[7] A. Alesanco and J. García, "Automatic real-time ECG coding methodology guaranteeing signal interpretation quality," IEEE Trans. Biomed. Eng., vol. 55, no. 11, pp. 2519-2527, Nov. 2008.

[8] A. Bilgin, M. W. Marcellin, and M. I. Altbach, "Compression of electrocardiogram signals using JPEG 2000," IEEE Trans. Consum Electron., vol. 49, no. 4, pp. 833-840, Nov. 2003.

[9] E. Kayacan, B. Ulutas, and O. Kaynuk, "Grey system theory-based models in time series prediction," Expert Syst. Appl., vol. 37, pp. 1784-1789, 2010.

[10] S. Lee, J. Kim, and M. Lee, "A real-time ECG data compression and transmission algorithm for an e-health device," IEEE Trans. Biomed. Eng., vol. 58, no. 9, pp. 2448-2455, Sep. 2011. 